\title{
LIF upregulates poFUT1 expression and promotes trophoblast cell migration and invasion at the fetal-maternal interface
}

\author{
S Liu ${ }^{1,4}$, J Wang ${ }^{1,4}$, H-M Qin², X-M Yan ${ }^{3}$, X-S Yang ${ }^{1}$, C Liu ${ }^{1}$ and Q Yan ${ }^{\star, 1}$
}

Trophoblast cell migration and invasion are crucial for the establishment of a successful pregnancy. Protein O-fucosyltransferases, such as poFUT1 and poFUT2, catalyze the O-fucosylation of proteins and have important roles in embryonic development. Leukemia inhibitory factor (LIF) is a critical cytokine in the regulation of embryonic development and implantation. However, the exact roles of poFUTs in embryo migration and invasion and the effects of LIF on the expression of poFUTs have not been studied in detail. In the current study, we showed that poFUT1 and LIF were highly expressed in human trophoblast cells and in the serum of women during the first trimester of a normal pregnancy. However, in patients with threatened abortion, poFUT1 and LIF levels were found to be reduced. There were no significant differences in the expression levels of poFUT2 between the two groups. The migration and invasion potential of trophoblasts in an explant culture and in an in vitro implantation model was decreased or increased upon altering poFUT1 expression levels by siRNA or cDNA transfection. Our results also revealed that LIF upregulated the expression of poFUT1. The upregulation of poFUT1 by LIF promoted trophoblast cell migration and invasion at the fetal-maternal interface by activating the PI3K/Akt signaling pathway. Taken together, these study findings suggest that poFUT1 may be used as a marker of embryo implantation.

Cell Death and Disease (2014) 5, e1396; doi:10.1038/cddis.2014.335; published online 28 August 2014

Human pregnancy is a complex biological process that requires synchrony between the develop embryo and the receptive uterine endometrium. The fertilized egg divides into a 2-, 4-, and 8-cell embryo before developing into the morula and then into the mature blastocyst. ${ }^{1,2}$ In the mature blastocysts, the inner cell mass (ICM), which comprises embryonic stem cells, differentiates into various tissues in the fetus. The trophoblast is the outer layer of embryonic tissue (also called villi) that facilitates the adhesion and penetration of the blastocysts into the endometrium during implantation. During the implantation of a human embryo, the progenitor trophoblast cells differentiate into the syncytiotrophoblast (STB) and the invasive trophoblast (iCTB) by different developmental pathways. The STB covers the villi and develops into the placenta, which mediates the exchange of nutrients, oxygen, and waste between maternal and fetal blood. The iCTB, which is derived from the trophoblast by epithelial-mesenchymal transition (EMT), forms cell columns and has a highly invasive character, causing it to migrate away from the attached embryo, invade the uterine epithelium and uterine spiral arteries to establish the maternal-fetal linkage. ${ }^{3-6}$ However, poor iCTB migration and invasion often results in a failure to establish the maternal-fetal connection and leads to abortion. Many factors at the maternal-fetal interface, such as hormones (e.g., progesterone), growth factors and their receptors (e.g., EGF and EGFR), and cytokines (e.g., leukemia inhibitory factor (LIF)), regulate trophoblast invasion. ${ }^{7-9}$ These molecules increase the invasion potential of the embryo by increasing the expression levels of matrix metalloproteinases (MMPs) and decreasing that of the tissue inhibitor of MMP (TIMP).

Protein glycosylation has an important role in many physiological and pathological processes, including inflammation, cancer metastasis, and embryo implantation. ${ }^{10-12}$ Protein fucosylation is one of the important types of glycosylation. It is classified into two kinds, $\mathrm{N}$-fucosylation and $O$-fucosylation, which are catalyzed by fucosyltransferases (FUTs) and protein $O$-fucosyltransferases (poFUTs), respectively. ${ }^{13,14}$ It is known that FUTs are expressed at specific stages of the menstrual cycle and are associated with reproductive function. ${ }^{15,16}$ Ponnampalam et al. ${ }^{17}$ reported an increased expression level of FUT4 in the secretory phase as compared with that observed in the proliferative phase. We had previously shown that FUT4 and FUT7, which are expressed in uterine epithelial cells, influence the adhesion potential of the embryo. ${ }^{18,19}$ poFUTs include poFUT1 and poFUT2. poFUT1 transfers L-fucose directly to the glycoproteins with epidermal growth factor (EGF)-like repeats. ${ }^{20}$ A mutation in poFUT1 caused embryonic lethality in mice; silencing poFUT1 led to abnormal development in

\footnotetext{
'Liaoning Provincial Core Lab of Glycobiology and Glycoengineering, Department of Biochemistry and Molecular Biology, Dalian Medical University, Dalian, People's Republic of China; ${ }^{2}$ Department of Pathology, The Secondary Affiliated Hospital of Dalian Medical University, Dalian, People's Republic of China and ${ }^{3}$ College of Medical Laboratory, Dalian Medical University, Dalian, People's Republic of China

*Corresponding author: Q Yan, Liaoning Provincial Core Lab of Glycobiology and Glycoengineering, Department of Biochemistry and Molecular Biology, Dalian Medical University, Dalian116044, People's Republic of China. Tel: + 86411 86110308; Fax: + 86411 86110308; E-mail: yanqiu63@ 126.com

${ }^{4}$ These authors contributed equally to this work.

Abbreviations: LIF, leukemia inhibitory factor; poFUT1, protein O-fucosyltransferase 1; ICM, inner cell mass; STB, syncytiotrophoblast; iCTB, invasive trophoblast; MMPs, matrix metalloproteinases; CK7, cytokeratin 7; TIMP, tissue inhibitor of MMP; EMT, epithelial-mesenchymal transition

Received 05.5.14; revised 02.7.14; accepted 03.7.14; Edited by A Finazzi-Agrò
} 
Drosophilas. ${ }^{20,21}$ poFUT2 catalyzes the O-fucosylation of the glycoproteins with thrombospondin type 1 repeats (TSRs). The disruption of poFUT2 in mice resulted in teratomas. ${ }^{22,23}$ However, the exact roles of poFUT1 and poFUT2 in human trophoblast migration and invasion have not been studied in detail.

LIF, a pleiotropic cytokine of the interleukin- 6 family, is essential for the successful completion of human pregnancy. ${ }^{24,25}$ LIF, secreted by both the trophoblast and the uterine endometrium, mediates embryonic development and implantation in an autocrine or paracrine manner at the maternal-fetal interface. In the mother, LIF level peaks during the secretory/postovulatory phase of the menstrual cycle and regulates uterine receptivity for blastocyst implantation. In the fetus, LIF promotes trophoblast proliferation, invasion, and differentiation. Reduced expression level of LIF led to incomplete embryonic development. A deficiency of LIF results in implantation failure and causes repeated abortions or unexplained infertility. ${ }^{26-29}$ However, the role of LIFmediated regulation of poFUT1 and poFUT2 in embryo implantation has not been elucidated.

In this study, we report that the levels of poFUT1 and LIF were higher in trophoblasts obtained from women with a normal pregnancy than those from patients with threatened abortion. However, the expression level of poFUT2 was not significantly different between the two groups. The poFUT1 promoted the migration and invasion of trophoblasts in an explant culture and in an in vitro implantation model. The upregulation of poFUT1 by LIF facilitated trophoblast cell migration and invasion through activating the PI3K/Akt signaling pathway.

\section{Results}

Expression of poFUT1 and LIF level was higher in women with normal pregnancy than in patients with threatened abortion. We analyzed the expression of poFUT1 and poFUT2 in villi of women in the first trimester of the normal pregnancy and threatened abortion by using immunohistochemistry (Figure 1A). Trophoblast cells were identified by cytokeratin 7 staining on a separate adjacent section (Figures 1Ac and d). The poFUT1 and poFUT2 could be detected in the villous trophoblast and the trophoblast column (TC) during the first trimester. In addition, the expression levels of poFUT1 were significantly lower in the villi of patients with threatened abortion than in the villi of women with normal pregnancy (Figures $1 \mathrm{Ae}$ and f). However, the expression levels of poFUT2 did not differ significantly between the two groups (Figures $1 \mathrm{Ag}$ and $\mathrm{h}$ )). The serum level of LIF in the normal pregnancy and threatened abortion groups was analyzed by enzyme-linked immunosorbent assay (ELISA) (Figure 1B). The results showed that the LIF level was significantly lower in the threatened abortion group $(5.08 \pm 1.79 \mathrm{ng} / \mathrm{l})$ than in the normal pregnancy group (10.60 $\pm 6.30 \mathrm{ng} / \mathrm{l} ; P<0.01)$.

Expression level of poFUT1 in trophoblast tissues and cells. Immunofluorescent staining and western blot analysis were used to confirm the expression of poFUT1 in the frozen sections of human villi and cultured trophoblast cells
A
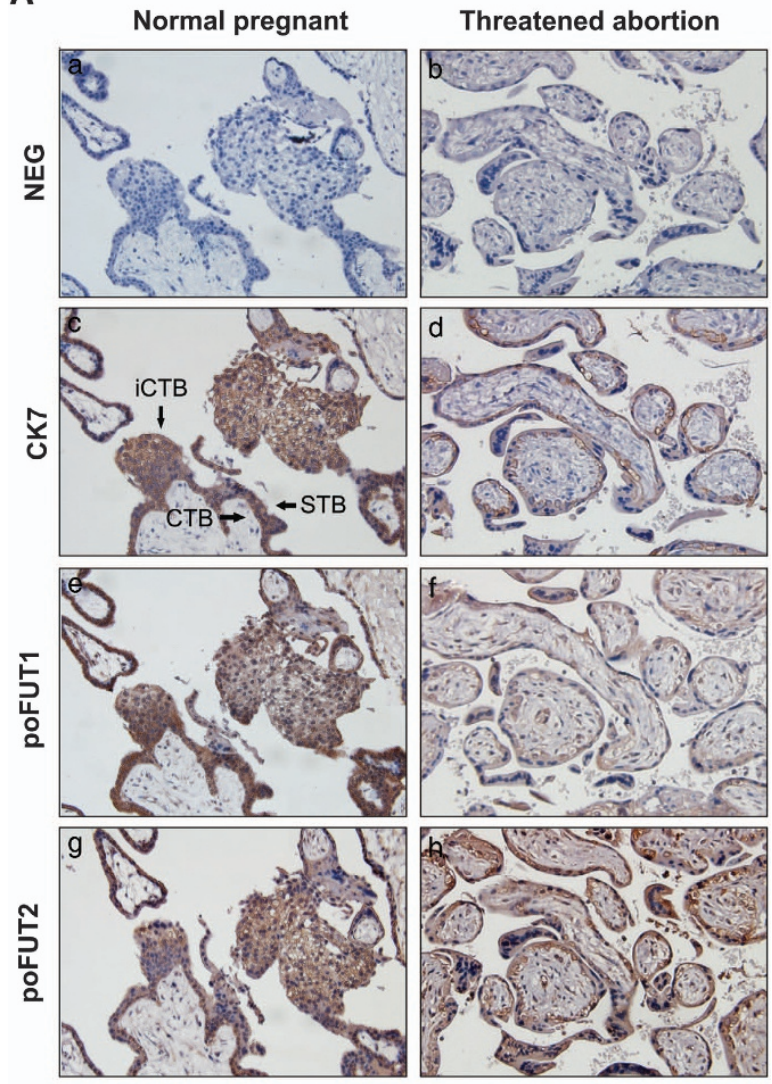

B

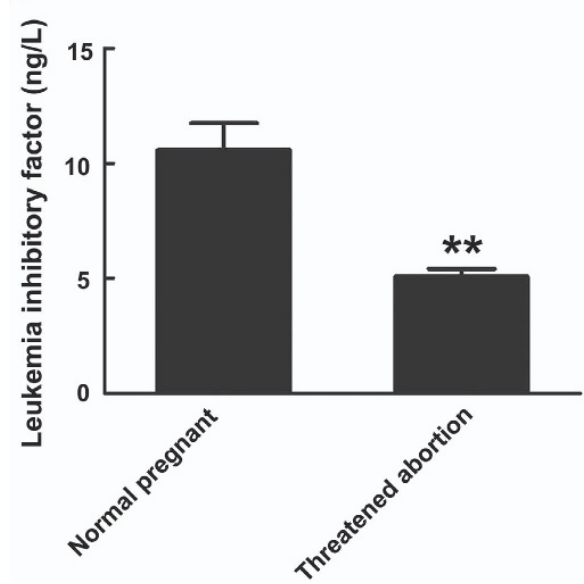

Figure 1 Expression of poFUT1 and LIF level in normal pregnant women and threatened abortion patients at first trimester. (A) Immunostaining of cytokeratin 7 (CK7) as a marker of CTB (c and d), poFUT1 (e and f), and poFUT2 (g and $h$ ) in normal pregnancy and threatened abortion villi from the first trimester. Negative control (NEG): serum IgG from rabbit was used in place of primary antibody ( $a$ and $b$ ). CTB: cytotrophoblast; STB: syncytiotrophoblast; iCTB: invasive cytotrophoblast. (B) The serum level of leukemia inhibitory factor (LIF) in normal pregnant woman $(n=30)$ and threatened abortion patients $(n=30)$ at the first trimester $\left({ }^{\star \star} P<0.01\right)$

(Figure 2). The fluorescent image showed that poFUT1 was expressed in human villi (Figure 2a). Immunofluorescent observation revealed that poFUT1 was expressed in JAR cells, where it was localized in the endoplasmic reticulum (Figure 2b). The results of the western blotting further 

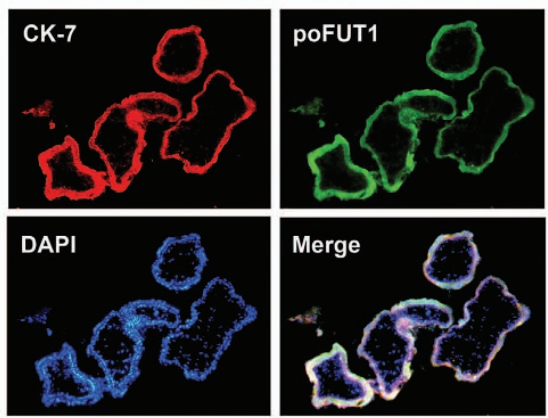

b
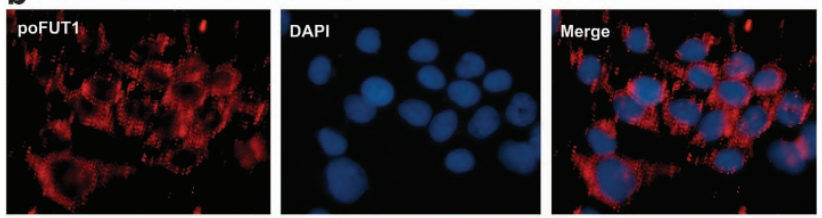

C

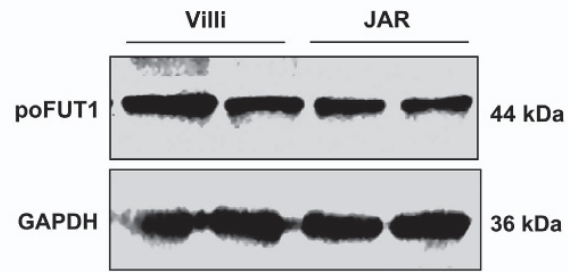

Figure 2 Expression of poFUT1 in trophoblast tissues and cells. (a) Representative immunofluorescence of poFUT1 in frozen sections in 7 weeks of first-trimester villi. Fluorescent signals specific to poFUT1 antibody were visualized as green, CK7 staining (red), and the nuclei staining with DAPI (blue). (b) Immunofluorescence of poFUT1 in trophoblast cells. Fluorescent signals specific to poFUT1 antibody were visualized as red, and the nuclei staining with DAPI (blue). (c) Expression of poFUT1 in villi and trophoblast cells (JAR) by western blotting

confirmed that both human villi and trophoblast cells express poFUT1 (Figure 2c).

Regulation of poFUT1 expression alters trophoblast outgrowth, migration, and invasion. To investigate the roles of poFUT1 in promoting implantation, the expression of poFUT1 was manipulated by transfecting the trophoblast cells with either siRNA to downregulate poFUT1 expression or CDNA transfection to upregulate poFUT1 expression. siRNAs (siRNA-1, -2, and -3) targeting poFUT1 were individually transfected into trophoblast cells. The expression levels of poFUT1 mRNA and protein were analyzed using real-time $\mathrm{PCR}$ and western blotting, respectively. The trophoblast cells transfected with siRNA-1 and siRNA-2 had significantly lower expression levels of poFUT1 mRNA and protein than those transfected with scramble RNA (CON siRNA), which was used as the control (Figures 3A and B).

Extravillous explants from first-trimester human villi were transfected with poFUT1 siRNA-1 or siRNA-2, and cultured for 24 and $48 \mathrm{~h}$ on matrigel-coated dishes. After $24 \mathrm{~h}$ of culture, while explants anchored on matrigel and started to outgrowth, no significant difference was observed between CON siRNA and poFUT1 siRNA-1 or siRNA-2 treated groups. At $48 \mathrm{~h}$ culture, however, poFUT1 siRNA-treated group showed a significant decreased outgrowth (Figures $3 \mathrm{Cb}$ ).
The successful transfection of siRNA into explant was determined by FITC-tagged CON siRNA, which exhibited green fluorescence (Figures $3 \mathrm{Ca}$ ). The migration and invasion potentials of the trophoblast cells transfected with poFUT1 siRNA-1 and siRNA-2 were evaluated by the transwell migration and matrigel invasion assays, respectively. Compared with the CON SiRNA, poFUT1 siRNAs significantly decreased the percentage of cells that showed migration (Figure 3D; ${ }^{* \star} P<0.01$ ) or invasion (Figure $3 \mathrm{E} ;{ }^{* \star} P<0.01$ ). The invasion potential of the trophoblast after poFUT1 siRNAs transfection was studied by assaying the activity of MMP-2 and the levels of TIMP-1 and -2 using gelatin zymography and western blotting, respectively. As illustrated in Figure 3F, the suspension medium from poFUT1 siRNA-treated cells exhibited much lower levels of MMP-2 activity than that from CON siRNA-treated cells. TIMP-1 and -2 levels were significantly higher in poFUT1 siRNA-transfected cells than in those transfected with control siRNA (Figure 3G).

However, transfection of trophoblasts cells with poFUT1 cDNA increased poFUT1 expression at both mRNA and protein levels, as detected by real-time PCR and western blot analysis (Figures $4 \mathrm{a}$ and $\mathrm{b}$ ). Moreover, the upregulation of poFUT1 facilitated cell migration (Figure $4 \mathrm{c}$; ${ }^{\star *} P<0.01$ ) and invasion (Figure $4 \mathrm{~d}$; ${ }^{\star \star} P<0.01$ ) compared with the mock control, as analyzed by transwell cell migration assay and matrigel cell invasion models. Upregulation of poFUT1 also increased MMP-2 activity, as determined by gelatin zymography (Figure 4e), and TIMP-1 and - 2 levels, as detected by western blotting (Figure 4f).

LIF upregulates poFUT1 expression in trophoblast cells and promotes trophoblast outgrowth and invasion. LIF is an essential factor for embryo implantation. To investigate whether LIF can regulate poFUT1 expression, trophoblast cells were treated with various concentrations of $\operatorname{LIF}(0,10$, and $50 \mathrm{ng} / \mathrm{ml}$ ) for different time intervals $(24,48$, and $72 \mathrm{~h}$ ). Protein samples were collected and poFUT1 levels were analyzed by western blotting. The results revealed that LIF increased expression levels of poFUT1 (Figures $5 a$ and b). The results also showed that LIF restored the reduced poFUT1 levels in poFUT1 siRNA-transfected trophoblast cells (Figure $5 c$ ), and further elevated the increased poFUT1 levels in poFUT1 cDNA-transfected trophoblast cells (Figure 5d).

To investigate whether the LIF-mediated expression of poFUT1 promoted trophoblast outgrowth and invasion, an extravillous explant culture and a matrigel cell invasion model were used. In Figure 6a, bright-field images (Figures 6Aa, f and $k$ ) showed that poFUT1 siRNA significantly decreased trophoblast outgrowth, while LIF restored the inhibition. Darkfield images showed the IF staining (Figures $6 \mathrm{Ab}-\mathrm{e}, \mathrm{g}-\mathrm{j}$, and $\mathrm{i}-0$ ). CK7 (red) is a marker for trophoblast cells (Figures 6Ab, j, and I). Compared with the group treated by CON SiRNA, poFUT1 siRNA was significantly decreased poFUT1 level (green) in trophoblast cells and LIF could restore the poFUT1 level (Figures 6Ac, h, and $\mathrm{m}$ ). In the matrigel cell invasion model, LIF also restored the trophoblast cell invasion potential. LIF was found to regulate the activity of MMP-2 and the expression of TIMP-1 and -2 (Figures $6 \mathrm{~A}$, $B$, and D). 
A

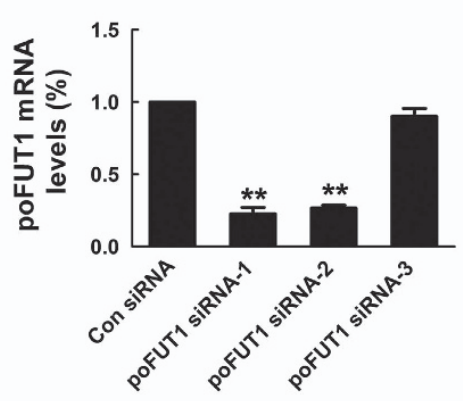

B

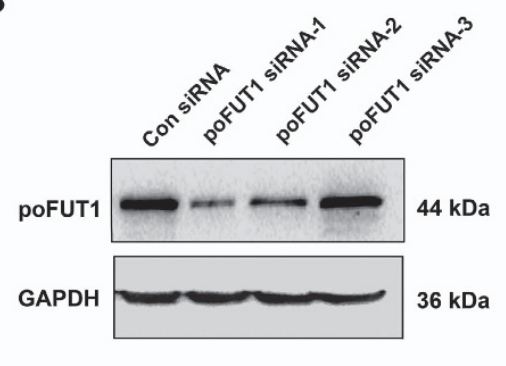

C

a

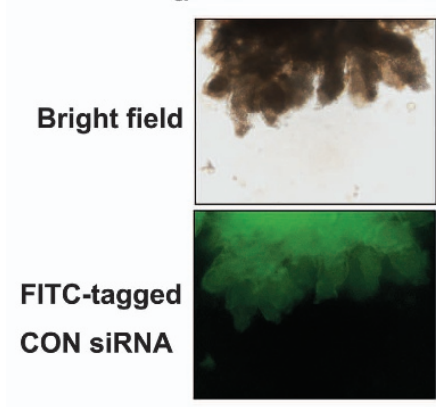

D
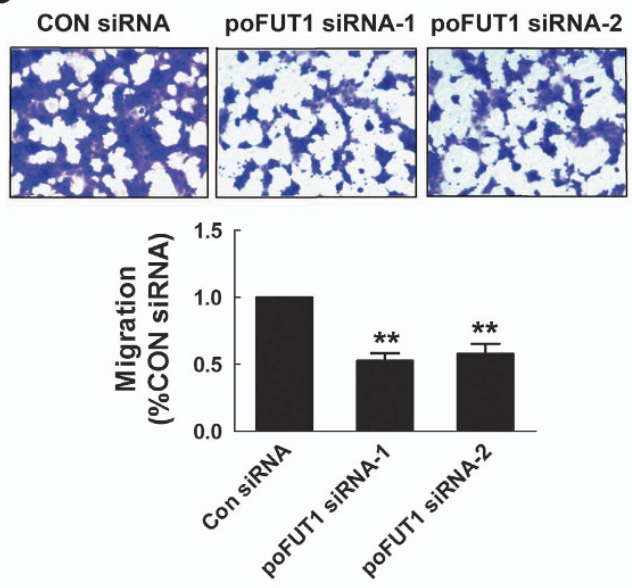

F

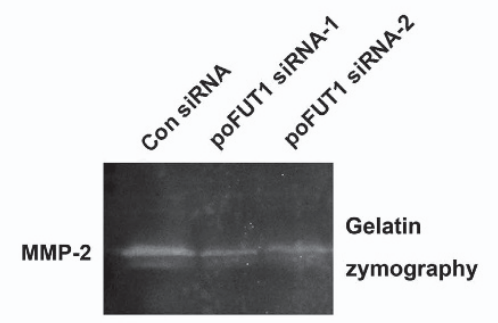

b

$24 \mathrm{~h}$
CON SIRNA

poFUT1 SIRNA1-1

poFUT1 siRNA-2
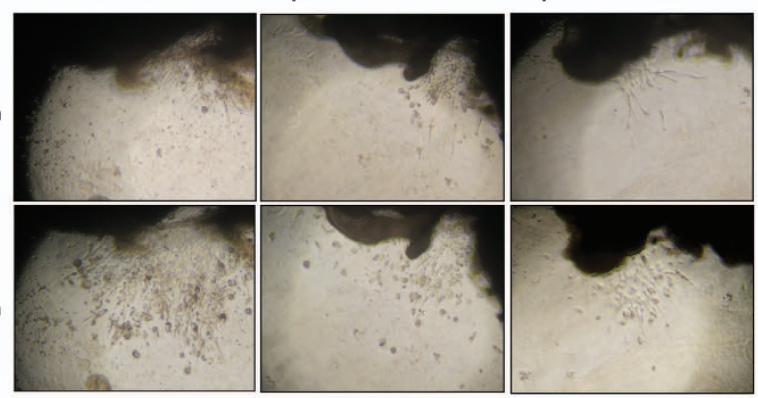

E
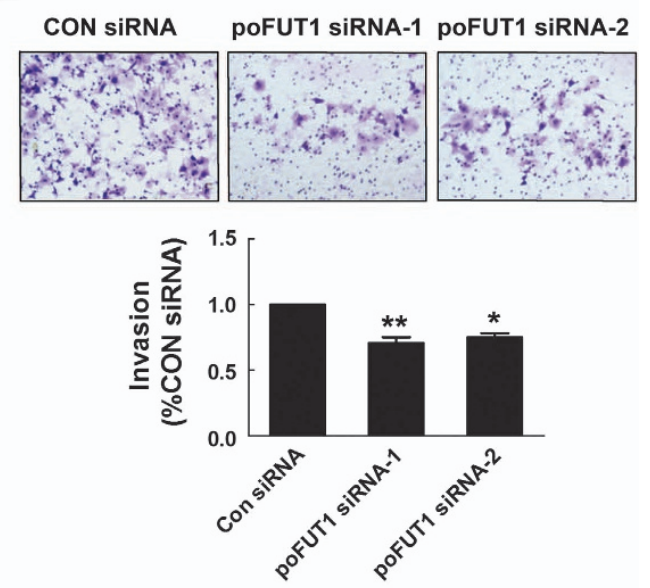

G

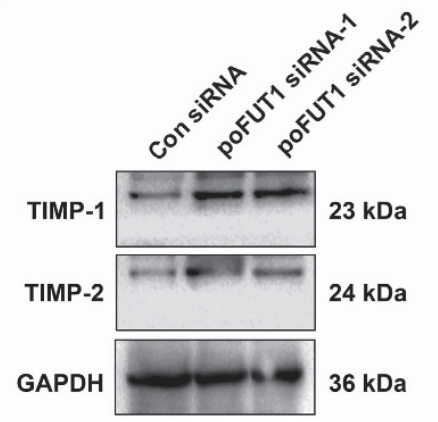

Figure 3 Silencing poFUT1 suppresses trophoblast outgrowth in extravillous explant cultures, migration and invasion of trophoblast cells. (A and B) Expression of poFUT1 in trophoblast cells after control (CON) or poFUT1 siRNA transfection by real-time PCR and western blotting. (C) (a) Villi transfected with FITC-tagged siRNA, showing the transfection efficiency. (b) Extravillous explants from 7 weeks of gestation maintained in culture on matrigel were incubated with CON siRNA or poFUT1 siRNA. Representative pictures of explants were taken under the microscope $(\times 10)$ after 24 and $48 \mathrm{~h}$ of culture in vitro. (D and E) Representative images of the cells treated with control (CON) or poFUT1 siRNA in transwell migration assay and matrigel invasion assay $(\times 100)$. The statistical bar graphs showed the summary of three independent experiments $\left({ }^{\star \star} P<0.01\right)$. (F) Gelatin zymography assay of MMP-2 in the culture medium collected from trophoblast cells which were treated with Con siRNA or poFUT1 siRNA-1, -2 . (G) Western blot analysis of TIMP-1 and TIMP-2 in trophoblast cells transfected with poFUT1 siRNA-1, -2 or Con siRNA 
a

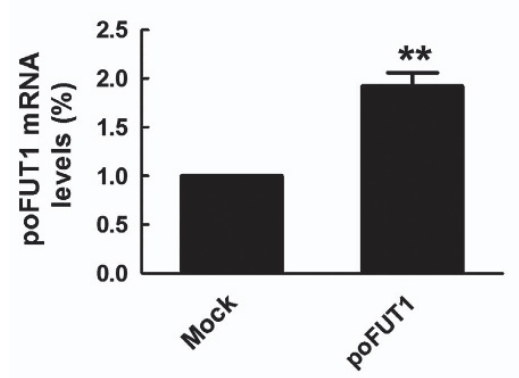

C
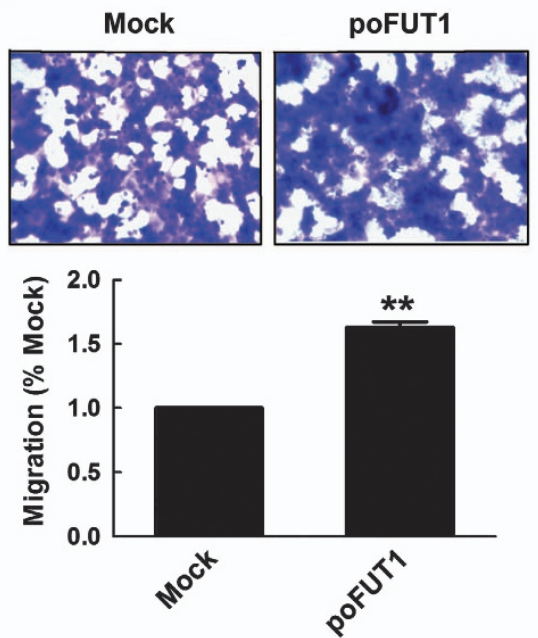

e

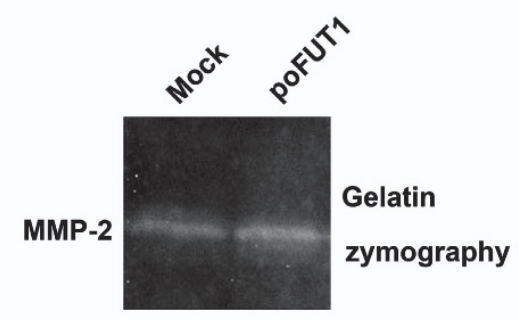

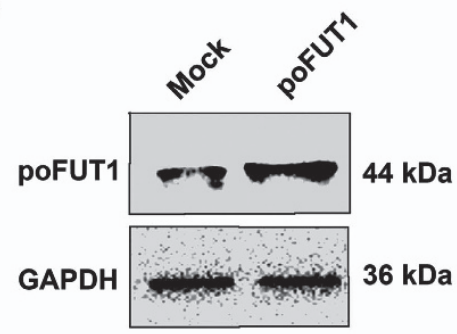

d


f

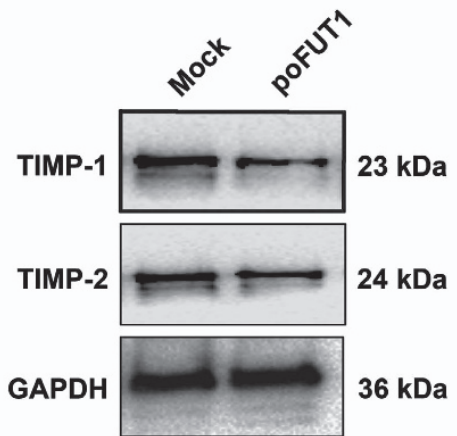

Figure 4 Overexpression of poFUT1 promotes migration and invasion of trophoblast cells. (a and $\mathbf{b}$ ) Expression of poFUT1 in trophoblast cells after poFUT1 cDNA transfection by real-time PCR and western blotting. (c and $\mathbf{d}$ ) Representative images of the cells transfected with poFUT1 cDNA in transwell migration assay and Matrigel invasion assay $(\times 100)$. The statistical bar graphs show the summary of three independent experiments $\left({ }^{* *} P<0.01\right)$. (d) Gelatin zymography assay of MMP-2 in the culture medium collected from trophoblast cells which were transfected with poFUT1 cDNA or Mock plasmid. (e) Western blot analysis of TIMP-1 and TIMP-2 in trophoblast cells transfected with poFUT1 cDNA or Mock plasmid

LIF further elevated the poFUT1 levels in poFUT1 cDNAtransfected trophoblast cells (Figure $5 d$ ), promoted trophoblast cell invasion, and regulated MMP-2 activity and TIMP-1 and -2 expression (Figures $6 \mathrm{C}$ and $\mathrm{E}$ ).

Upregulation of poFUT1 by LIF promotes cell invasion through the activation of the PI3K/Akt signaling pathway. To explore the molecular mechanism underlying the LIF-mediated regulation of poFUT1 in trophoblast invasion, the activation of the PI3K/Akt signaling pathway was analyzed. Trophoblast cells were pretreated with poFUT1
SiRNA, LIF, or LY294002 (an inhibitor of PI3K), and the presence PI3K/Akt signaling molecules, p-PDK, p-Akt (468), p-Akt (308), was detected by western blotting (Figure 7).

The results showed that silencing poFUT1 resulted in the inhibition of p-PDK, p-Akt(468), and p-Akt(308) expression in the PI3K/Akt signaling pathway (Figure 7a). An invasion assay confirmed that poFUT1 siRNA transfection decreased cell invasion rate in matrigel cell invasion models, inhibited MMP-2 activity, and increased TIMP-1 and -2 expression (Figures 7c and e). LIF could partly restore decreased cell invasion rate by activating the PI3K/Akt signaling pathway. 


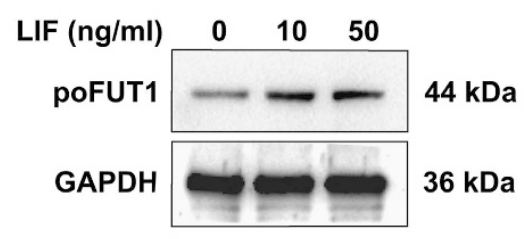

C

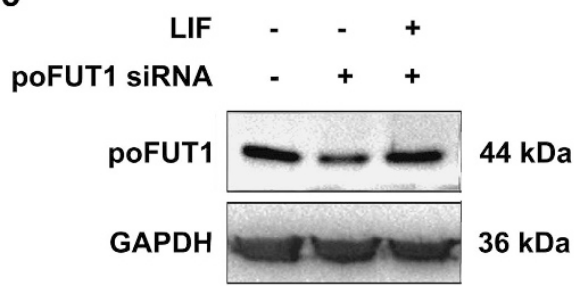

b

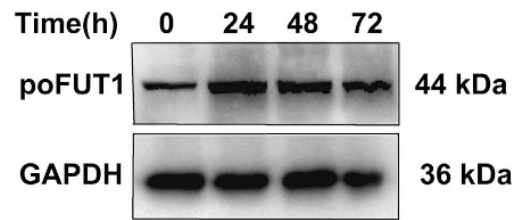

d

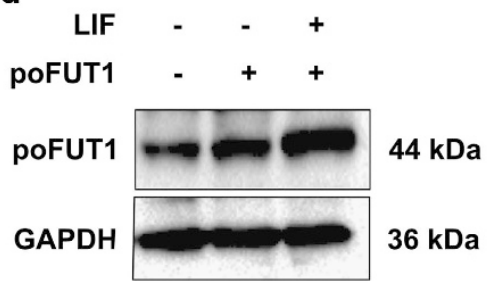

Figure 5 Leukemia inhibitory factor (LIF) upregulates poFUT1 expression in trophoblast cells. (a) Effects of LIF (0, 10, and $50 \mathrm{ng} / \mathrm{ml})$ on poFUT1 expression in trophoblast cells by western blotting. (b) poFUT1 expression in trophoblast cells treated with LIF (10 ng/ml) for 24, 48, or $72 \mathrm{~h}$. (c and d) LIF regulation of poFUT1 expression in trophoblast cells transfected with poFUT1 siRNA or poFUT1 cDNA

Moreover, addition of PI3K/Akt inhibitor suppressed the invasion potential of the trophoblast cells (Figures 7c and e).

The poFUT1 cDNA transfection increased the expression levels of p-PDK, p-Akt(468), and p-Akt(308) and activated the $\mathrm{PI} 3 \mathrm{~K} /$ Akt signaling pathway, which in turn promoted trophoblast invasion (Figures 7b, d, and f).

\section{Discussion}

Embryo adhesion, migration, and invasion are regulated in a strict spatiotemporal manner. A variety of molecules, which are expressed at specific stages of the menstrual cycle, have important roles in the embryo implantation process, such as hormones, growth factors, growth factor receptors, and cytokines. ${ }^{7-9,30,31}$ Abnormal expression level of these factors may lead to the impaired function of the downstream molecules, which in turn may eventually result in infertility, abortion, or pregnancy-related complications. Glycosyltransferases catalyze protein glycosylation, which is involved in regulating the proliferation, migration, and invasion potential of the trophoblast. For example, polysialyltransferase IV (ST8SialV), which enhances trophoblast migration and invasion, is highly expressed in trophoblast cells during the first trimester and downregulated gradually during the course of the pregnancy. ${ }^{32}$ Yamamoto et al. ${ }^{33}$ reported that silencing $\mathrm{N}$-acetylglucosaminyltransferase $\mathrm{V}(\mathrm{GnT}-\mathrm{V})$ inhibited the migration and invasion of human cytotrophoblasts. FUTs also have important roles during embryo implantation. FUT7 is highly expressed in both mature blastocysts in mice and chorionic tissues in humans, as well as in the embryonic trophoblast cells. The downregulation of FUT7 expression by antisense oligodeoxynucleic acid decreased the embryo adhesion rate in an in vitro implantation model. ${ }^{18,19}$ In our previous studies, we found that even after $\mathrm{N}$-linked sugar chains were deleted by treatment with $N$-tunicamysin and PNGase, many fucosylated proteins could be detected in trophoblast cells in a UEA lectin blot (unpublished data). Moreover, embryo implantation was not completely inhibited in these experiments. O-fucosylation has an important role in embryo development and culmination. Therefore, we hypothesized that $O$-fucosylation is also closely related to embryo implantation. $O$-fucoslyation is catalyzed by poFUT1 and poFUT2. In this study, we analyzed the expression of poFUT1 and poFUT2 in the villi of women with normal pregnancy and in patients with threatened abortion in the first trimester of pregnancy using immunohistochemistry. The results revealed that poFUT1 was highly expressed in the villi of women with normal pregnancy. However, villi from patients with threatened abortion showed decreased poFUT1 levels (Figure 1A). Moreover, silencing poFUT1 not only significantly inhibited trophoblast outgrowth in the extravillous explant culture model, but also suppressed the migration and invasion potentials of cultured trophoblast cells (Figure 3). To corroborate our hypothesis that decreased POFUT1 level may increase the risk of abortion. Serum LIF level was also simultaneously analyzed. It has been reported that LIF stimulates the proliferation, differentiation, and invasion of extravillous trophoblast explants and trophoblast cell lines. ${ }^{34}$ A mutation or deficiency in LIF is associated with embryo dormancy and pregnancy loss. Reduced levels of LIF increase the risk of miscarriage in patients undergoing in vitro fertilization. ${ }^{35,36}$ In this study, we found that as compared with the normal pregnancy group, the threatened abortion group had significantly diminished levels of serum LIF (Figure 1B). This result is consistent with the observation that poFUT1 level was reduced in patients with threatened abortion. Therefore, poFUT1, together with LIF, may be a novel marker of threatened abortion and may be useful for the evaluation of embryonic development.

LIF is an upstream regulatory factor. LIF activates embryo and promotes embryo implantation by regulating the expression levels and functions of specific molecules. For example, LIF decreases embryonic lethality by elevating SOCS3 expression, ${ }^{37}$ increases PAPPA level which is associated with embryonic development, ${ }^{38}$ and promotes embryo adhesion to the uterine epithelium by increasing the levels of integrin $\beta 3 .^{39}$ LIF is also involved in immune tolerance through the regulation of HLA-G, a class I MHC molecule specifically 
A

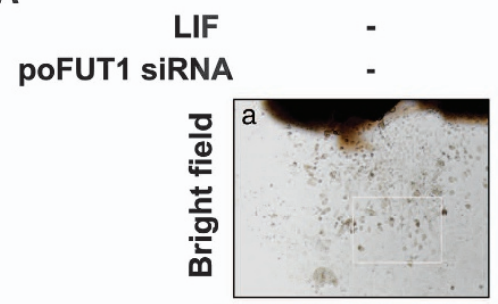

$+$
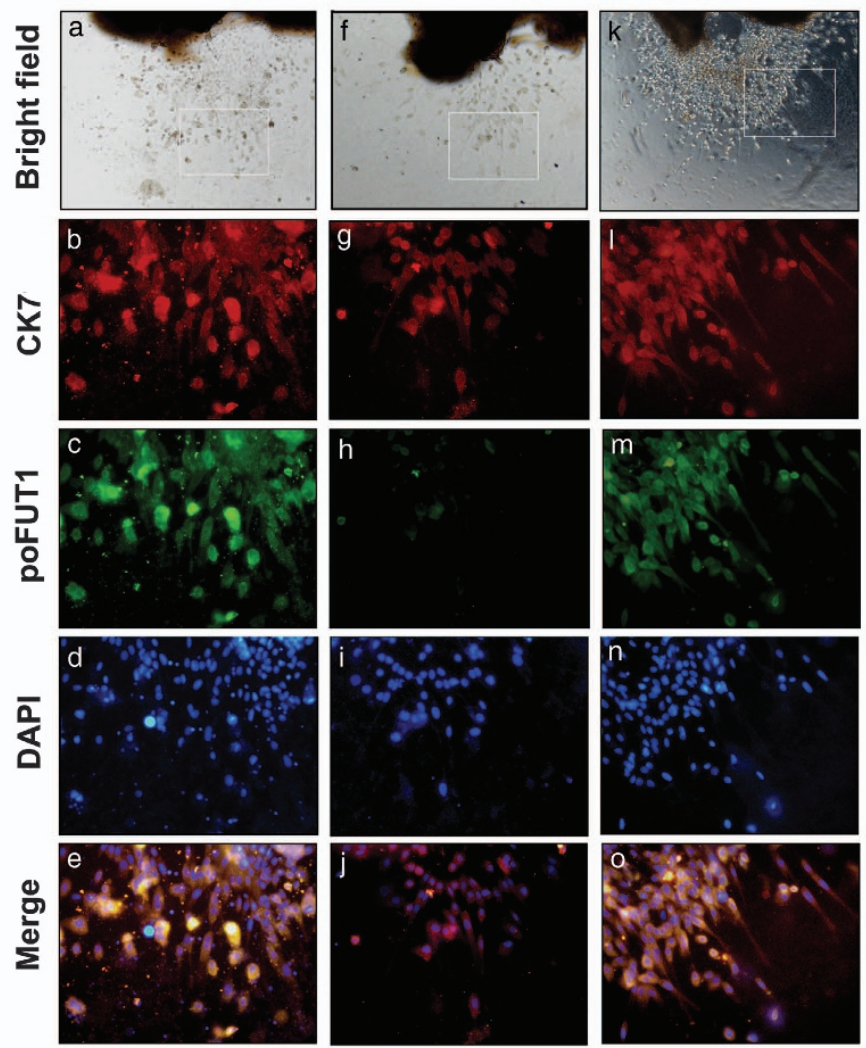

B

C
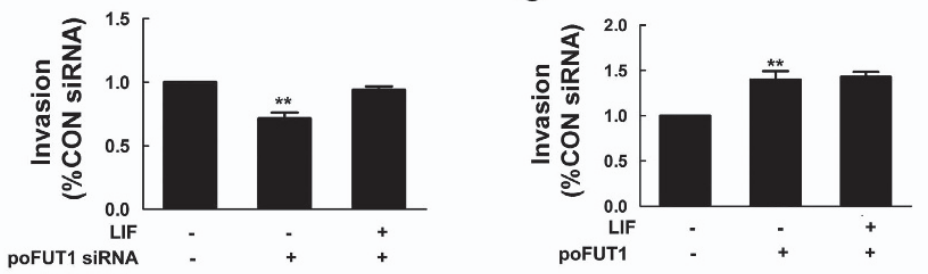

D

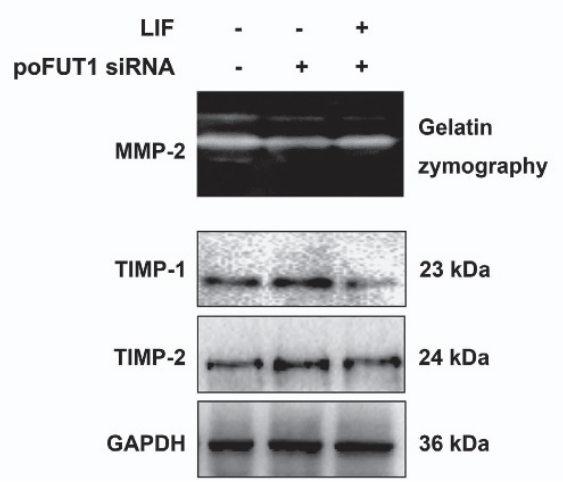

$\mathbf{E}$

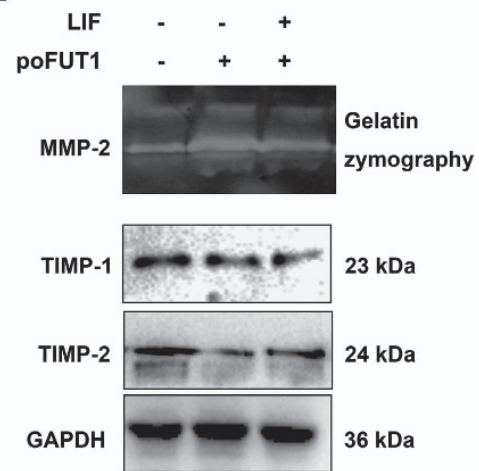

Figure 6 LIF facilitates trophoblast outgrowth in extravillous explant cultures, migration and invasion of trophoblast cells through poFUT1. (A) Effect of LIF on outgrowth of trophoblast transfected with poFUT1 siRNA using extravillous explant culture. Whole mount immunofluorescent assay indicating the silencing of poFUT1 in the outgrowing. Bright-field images $(a, f$, and $k)$ show the outgrowth of the explants. The boxed areas in $(a, f$, and $k)$ are enlarged and shown in $(b-d$, gi, l-n), respectively $(\times 40)$. The antibodies used for immunofluorescent staining indicated on the images. (B and C) Effect of LIF on invasion in poFUT1 siRNA or poFUT1 cDNA-transfected trophoblast cells. (D and E) Effect of LIF on MMP-2 activity by gelatin zymography and TIMP-1 and -2 expression by western blotting in poFUT1 siRNA or poFUT1 cDNA-transfected trophoblast cells 
a

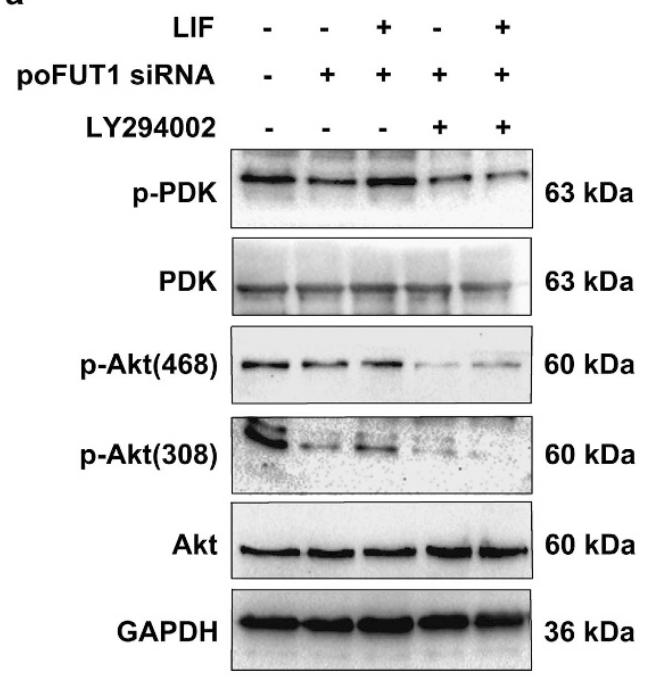

c

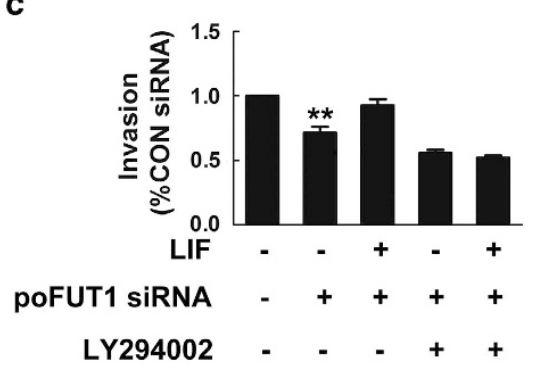

e

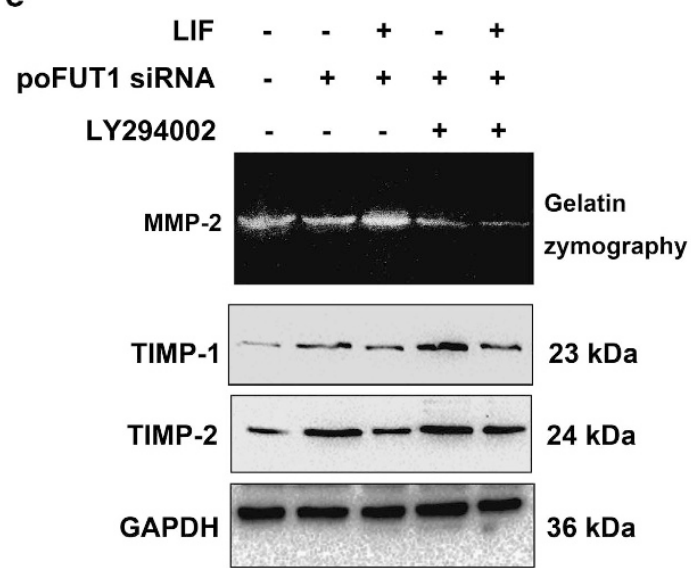

b

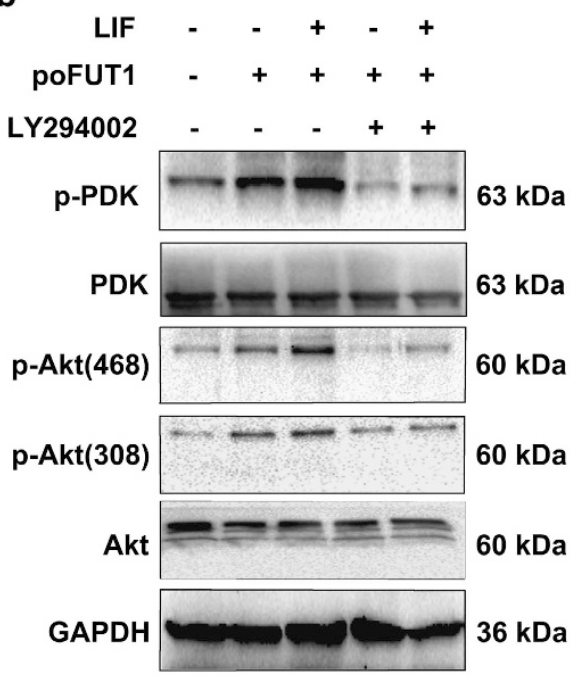

d

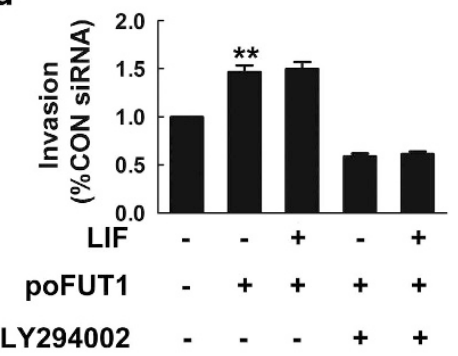

f
LY294002 - - $\quad+\quad+$

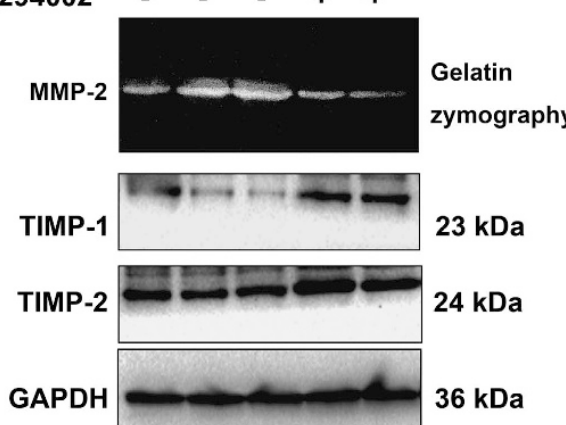

Figure 7 Upregulation of poFUT1 by LIF promotes cell migration and invasion through PI3K/Akt signal pathway. (a) Trophoblast cells were pretreated by poFUT1-siRNA, LIF or inhibitor of PI3K (LY294002), and activation of PI3K/Akt signal molecules (p-PDK, p-Akt468, and p-Akt308) was measured by western blotting. (c and e) Invasion potential and MMP-2 activity and TIMP-1, -2 expression were detected. (b) Trophoblast cells pretreated by poFUT1 cDNA, LIF or inhibitor of PI3K, and activation of PI3K/Akt signal molecules was measured by western blotting. ( $\mathbf{d}$ and $\mathbf{f}$ ) Invasion potential and MMP-2 activity and TIMP-1, -2 expression were detected

expressed by invasive cytotrophoblast cells. ${ }^{40}$ To the best of our knowledge, the role of LIF in poFUT1 regulation has not been reported to date. In our previous study, we found that LIF treatment upregulated the expression of FUT7, which has a role in embryo adhesion, in mouse embryos. ${ }^{41}$ In this study, trophoblast cells were used to analyze the regulatory effect of LIF on poFUT1. The results showed that LIF treatment upregulated the expression of poFUT1 in trophoblast cells
(Figure 5). LIF also restored trophoblast outgrowth potential in a poFUT1 siRNA-transfected extravillous explant (Figure 6A). Consistent with this result, LIF could partially restore poFUT1 expression and invasion potential in poFUT1-silenced trophoblast cells (Figures 5 and 6 ). These results suggest that poFUT1 is one of the target molecules regulated by LIF.

Embryo invasion is a key step in the implantation process. The trophoblast secretes MMPs, which degrade the ECM. 
This facilitates blastocyst invasion through the decidua and into the maternal vasculature. ${ }^{42}$ MMP-2 and TIMPs are associated with cell invasiveness and metastasis potential. MMP-2 has been implicated in the remodeling of the ECM during the trophoblast invasion process. ${ }^{43,44}$ In this study, we found that silencing or overexpressing poFUT1 could downregulate or upregulate trophoblast invasion by altering the activity of MMP-2 (Figures 3F and 4e). In addition, in the poFUT1-silenced group, LIF increased the MMP-2 activity, further enhancing trophoblast invasion (Figure 6D). TIMPs, which are the major endogenous inhibitors of MMP activity in tissues, inhibit trophoblast invasion. ${ }^{45}$ In this study, poFUT1 siRNA increased TIMP-1 and TIMP-2 expression, further inhibited MMP-2 activity, and decreased trophoblast invasion (Figure 3G).

The present study also investigated the molecular mechanism by which LIF and poFUT1 regulates trophoblast invasiveness. LIF regulates embryo development and implantation through activating many signaling pathways. For example, LIF regulates trophoblast differentiation through activating the JAK/STAT and MAPK signaling pathways. ${ }^{46}$ LIF maintains pluripotency in mouse and primate embryonic stem cells by activating the PI3K/Akt signaling pathway. ${ }^{47,48}$ Activation of the PI3K/Akt signaling pathway was also reported to promote the metastasis ability and invasiveness of many tumors by enhancing the activity of MMPs. ${ }^{49,50}$ To a certain extent, trophoblast invasion in reproduction is similar to that of the tumor metastasis. Therefore, we hypothesized that trophoblast invasion promoted LIF correlated with activation of PI3K/Akt signaling pathway. However, the mediating molecules involving in this process remain not clear. The poFUT1 is a key enzyme in regulating the $O$-fucosylation of specific glycoproteins on the cell surface and the downstream signaling pathways, such as notch. Shi et al. ${ }^{20}$ reported that poFUT1 altered fucosylation of notch receptor could affect notch signaling and embryo development, but the exact signaling pathway remains not clear. In this study, silencing poFUT1 inhibited the activation of the signaling molecules ( $\mathrm{p}-\mathrm{PDK}, \mathrm{p}-\mathrm{Akt} 468$, and p-Akt308) in $\mathrm{PI} 3 \mathrm{~K} /$ Akt signaling pathway, which decreased the cell invasion potential (Figures 7a, c, and e). Whereas, the addition of LIF in the poFUT1 silencing group could upregulate the expression of poFUT1 (Figure 5c), and restore the activation of PI3K/Akt signaling pathway, as well as increase trophoblast cell invasion potential (Figure 7). The above results indicate that LIF upregulates poFUT1 expression, which further activates PI3K/Akt signaling pathway and promotes trophoblast invasion. Although our results have demonstrated that the upregulation of poFUT1 by LIF promotes embryo invasion through the activation of the PI3K/AKT signaling pathway, future investigation is required to explore the target $O$-fucosylated glycoproteins catalyzed by poFUT1 in PI3K/Akt signaling pathways.

In summary, to our knowledge, this study is the first to demonstrate that poFUT1 promotes trophoblast invasion and migration at the maternal-fetal interface. LIF also promotes trophoblast cell invasion and migration by upregulating the expression of poFUT1. Moreover, the upregulation of poFUT1 by LIF promotes cell invasion and migration through the PI3K/AKT signaling pathway. The correlation between the decreased expression of poFUT1 and abortion provides a pathological criterion that may be applied for the diagnosis and treatment of threatened abortion.

\section{Materials and Methods}

Serum and tissue sample. The protocols for human study were in accordance with the Institutional Review Board of Dalian Medical University. Samples were obtained from Yingkou Central Hospital and the Secondary Affiliated Hospital of Dalian Medical University from 2012 to 2013. Women serum samples used in this study were obtained from the women at the ages of 25-35. The non-pregnant control group $(n=30)$ was excluded from other gynecological abnormalities. The pregnant women were confirmed by ultrasound detection at 6-10 gestational weeks. The serum samples collected from normal pregnant group $(n=30)$ and threatened abortion group $(n=30)$ were used to analyze LIF level. These paraffin-embedded human villi were used for keratin 7 (CK-7) and poFUT1/2 immunohistochemical and immunofluorescent staining. The fresh human villi tissues were collected from the non-drug abortion women.

Cell culture. The human embryonic JAR cells were obtained from the American Type Culture Collection (Manassas, VA, USA). The JAR cells were maintained in EMEM/F12 (Invitrogen, Carlsbad, CA, USA) supplemented with $10 \% \mathrm{FBS}, 100 \mathrm{U} / \mathrm{ml}$ penicillin, and $100 \mu \mathrm{g} / \mathrm{ml}$ streptomycin at $37^{\circ} \mathrm{C}$ under $5 \% \mathrm{CO}_{2}$ in humidified air according to standard procedures. The growth medium was renewed every 2-3 days.

ELISA. Commercial ELISA kits (Rocho, Norcross, GA, USA) were used to detect the serological values of LIF according to the manufacturer's instructions. The absorbance was measured at $450 \mathrm{~nm}$ using an ELISA microplate reader (Thermo Fisher Scientific, Waltham, MA, USA). Three samples were tested in each group for each time.

Immunohistochemistry and immunofluorescence. Tissue slides were deparaffinized and rehydrated. Endogenous peroxidase activity was blocked with $0.3 \%$ hydrogen peroxide for $15 \mathrm{~min}$. Slides were blocked with $10 \%$ normal goat serum for $10 \mathrm{~min}$, and incubated with anti-human poFUT1/2 antibody (1:50; Protein Tech, Chicago, IL, USA) and anti-human keratin 7 antibody (1:50, Protein Tech) at $4{ }^{\circ} \mathrm{C}$ for $12 \mathrm{~h}$. The slides were incubated with biotinylated secondary antibody at $37^{\circ} \mathrm{C}$ for $15 \mathrm{~min}$, and reacted with a streptavidin-peroxidase conjugate at $37^{\circ} \mathrm{C}$ for $10 \mathrm{~min}$, then used $3,3^{\prime}$-diaminobenzidine as a chromogen substrate. Meyer's hematoxylin was used as a counterstained dye. A negative control was obtained by replacing the primary antibody with phosphate-buffered saline (PBS). Images were captured with the Olympus fluorescence microscope (Olympus, Tokyo, Japan).

Frozen sections were fixed with $4 \%$ paraformaldehyde for $48 \mathrm{~h}$ at room temperature, and then treated with $20 \%$ sucrose for $48 \mathrm{~h}$. Tissues were embedded with OCT and sliced to $18 \mu \mathrm{m}$ at $-20^{\circ} \mathrm{C}$. After blocking with goat serum for $2 \mathrm{~h}$ at room temperature, the primary anti-human poFUT1 antibody $(1: 50)$ and antihuman keratin $7(1: 50)$ were incubated at $4{ }^{\circ} \mathrm{C}$ for $12 \mathrm{~h}$. The next day, after $1 \mathrm{~h}$ incubation with phycoerythrin (PE)-conjugated anti-goat IgG (1:200; Santa Cruz Biotechnology, Santa Cruz, CA, USA) at $37^{\circ} \mathrm{C}$, slides were washed three times for 5 min with PBS, and then treated with DAPI (Santa Cruz Biotechnology) for 5 min at $37^{\circ} \mathrm{C}$. The poFUT1 and keratin 7 immunofluorescent staining was photographed with the inverted microscope (Olympus).

Explant culture and whole mount immunofluorescence staining. The explant culture was performed as described previously. ${ }^{51}$ In brief, small pieces of tissues $(2-3 \mathrm{~mm})$ from tips of first-trimester human placental villi (7 weeks) were dissected and explanted in Millicell-CM culture dish inserts $(0.4 \mathrm{~mm}$ pore size, Millipore, Carrigtwohill, Co., Cork, Ireland) pre-coated with phenol red-free matrigel substrate (Becton Dickinson, Bedford, MA, USA). Inserts were placed into 24-well culture dishes (Costar, Cambridge, MA, USA). The explants were cultured in serum-free DMEM/F12 media with $100 \mathrm{U} / \mathrm{ml}$ penicillin and $100 \mu \mathrm{g} / \mathrm{ml}$ streptomycin at $3 \% \mathrm{O}_{2} / 5 \% \mathrm{CO}_{2} / 92 \% \mathrm{~N}_{2}$. Trophoblast cell sprouting and migration from the distal end of the villous tips were recorded for 24 and $48 \mathrm{~h}$. To test the effect of poFUT1 on the trophoblast cell migration, siRNAs specifically targeting poFUT1 $(500 \mathrm{nM})$, an equal concentration of the control siRNA and LIF was introduced into wells of culture media. All explant experiments with cultured villi were repeated three times.

Whole mount immunofluorescent staining was performed to confirm the role of poFUT1 siRNA and LIF on trophoblast outgrowth. The explants cultured for $48 \mathrm{~h}$ together with matrigel were fixed by $4 \%$ neutral PFA at room temperature for $30 \mathrm{~min}$, and then washed $3 \times 30$ min in PBS with $1 \%$ Triton X-100. The explanted villi were blocked with goat serum for $1 \mathrm{~h}$, and then incubated with primary anti-human 
poFUT1 antibody $(1: 50)$ and anti-human keratin $7(1: 50)$ at $4{ }^{\circ} \mathrm{C}$ for 2 days. The explants were washed $3 \times 1 \mathrm{~h}$ in PBS with $1 \%$ Triton X-100 and $10 \% \mathrm{FBS}$, and $3 \times 10 \mathrm{~min}$ in PBS with $1 \%$ Triton $\mathrm{X}-100$. Explants were then incubated with fluorescent secondary antibody for 1 day and DAPI for $1 \mathrm{~h}$ sequentially. Finally, the fluorescent signals were photographed with the inverted microscope (Olympus). ${ }^{30}$

Real-time PCR. Cells were treated with RNAiso Plus reagent (Takara, Liaoning, China) for RNA extraction, and PrimeScript RT reagent Kit with gDNA Eraser kit (Takara) was used for synthesizing cDNA. SYBR Premix Ex Taq (Takara) was used for quantitative real-time PCR. Primers were as follows: poFUT1: 5'-CAGCGAAGCCCAGATAAGAAG-3' (forward), and 5'-CTGTAGGA AGCACTGAAGGAAATG-3' (reverse). GAPDH: 5'-GCACCGTCAAGGCTG AGA AC-3' (forward), and 5'-TGGTGAAGACGCCAGTGGA-3' (reverse). The reactions were performed with Applied Biosystems 7500 Fast Real-time PCR System (Life Technologies, Carlsbad, CA, USA).

Transfection of poFUT1 siRNA and poFUT1 overexpression plasmid. PoFUT1 siRNA and non-specific siRNA were dissolved in RNase-free water provided by the manufacturer to a stock concentration of $20 \mathrm{mM}$. Cells were plated into $60-\mathrm{mm}$ culture dishes and then transfected with $40 \mathrm{nM}$ of siRNA using lipofectamine 2000 following the manufacturer's instructions. poFUT1 overexpression plasmid PEX-3poFUT1 (GenePharma, Shanghai, China) was constructed and transfected into cells using lipofectamine 2000 following the manufacturer's instructions. All assays were performed $48 \mathrm{~h}$ after transfection. PoFUT1 siRNA sequences were as follows: poFUT1 siRNA-1 (688) sense $5^{\prime}$-GGUCAGACGAAAUGGUGAATT-3', antisense $5^{\prime}$-UUCA CCAUUUCGUCUGACCTT-3'; poFUT1 siRNA-2 (962): sense 5'-GGUCUACGUUG CUACUGAUTT-3', antisense 5'-AUCAGUAGCAACGUAGACCTT-3', PoFUT1 siRNA-3 (1089): sense 5'-GACCACUUUAUUGGCAACUTT-3', antisense $5^{\prime}$-AGUUGCCA AUAAAGUGGUCTT-3'.

Western blotting. To prepare whole-cell extracts, cells at $90 \%$ confluence were washed in PBS before incubation with lysis buffer (1\% Triton X-100, $150 \mathrm{mM}$ $\mathrm{NaCl}, 10 \mathrm{mM}$ Tris, pH 7.4, $1 \mathrm{mM}$ EDTA, $1 \mathrm{mM}$ EGTA, pH 8.0, $0.2 \mathrm{mM} \mathrm{Na}_{3} \mathrm{VO}_{4}$, $0.2 \mathrm{mM}$ phenylmethylsulfonyl fluoride and $0.5 \%$ Nonidet $\mathrm{P}-40$ ) on ice for $10 \mathrm{~min}$. The cell lysates were clarified by centrifugation at $9000 \times g$ for $10 \mathrm{~min}$, and the supernatants were collected. Protein concentration was determined with the Coomassie Protein Assay Reagent using bovine serum albumin as a standard. Cell lysates $(50 \mu \mathrm{g})$ were separated by $10 \%$ SDS-PAGE min-gel. Samples were transferred electrophoretically onto nitrocellulose membranes, blocked with TTBS containing $5 \%$ fat-free dry milk for $2 \mathrm{~h}$ and incubated for $3 \mathrm{~h}$ with the appropriate primary antibodies at the dilutions recommended by the suppliers. After incubation with an HRP-conjugated anti-goat secondary antibody, immunoreactive proteins were visualized with ECL detection system. Western blots shown are representative of at least three independent experiments. Densitometry of each band for the target protein was quantified by densitometry analysis with Labworks 4.6 (Media Cybernetics, Inc, Bethesda, MD, USA). The protein band intensity was quantified by the mean \pm S.D. of three experiments for each group, as determined from densitometry relative to $\beta$-actin.

Matrigel cell invasion and transwell cell migration assay. For matrigel cell invasion assay, transwell inserts $(6.5 \mathrm{~mm}$, Costar) containing polycarbonate filters with $8 \mathrm{~mm}$ pores were precoated with $50 \mathrm{ml}$ of $1 \mathrm{mg} / \mathrm{ml}$ matrigel matrix (Becton Dickinson). For cell migration assay, the inserts were not precoated with matrigel. In all, $1.0 \times 10^{5}$ of JAR cells in serum-free medium were plated in the upper chamber, whereas medium with $10 \%$ FBS was added to the lower chamber. After incubating for $24 \mathrm{~h}$, the cells on the matrigel side of the inserts were removed by cotton swab. The inserts were fixed in methanol and stained with crystal violet. The number of invaded or migrated cells attached to the other side of the insert was counted under a light microscope (Olympus) in five random fields at a magnification of $\times 100$. Three independent experiments were performed. Numbers of invasive or migrated cells under different treatments were normalized to the control and expressed as a means of invasion or migration percentage $(\%) \pm$ S.D.

Gelatin zymography assay. MMP-2 activity was detected using the gelatin zymography assay. The supernatants were collected and used as the samples. Then, $10 \mu \mathrm{l}$ total protein was electrophoresed on $8 \%$ SDS-polyacrylamide gels copolymerized with $1 \%$ gelatin. After electrophoresis, the gels were washed five times in $2.5 \%$ Triton X-100 (20 min each) and two times in buffer without Triton
$\mathrm{X}-100$ to remove Triton $\mathrm{X}-100$, and then incubated in $50 \mathrm{mmol} / \mathrm{I}$ Tris-Cl, $\mathrm{pH} 7.6$, and $5 \mathrm{mmol} / \mathrm{l} \mathrm{CaCl} 2\left(18 \mathrm{~h}, 37^{\circ} \mathrm{C}\right)$. The gels were stained with $0.1 \%$ Coomassie blue R250 and destained in 10\% isopropanol and 10\% acetic acid in $\mathrm{H}_{2} \mathrm{O}$. MMP-2 was detected as transparent bands on the blue background of a Coomassie bluestained gel.

Statistical analysis. The quantitative data derived from three independent experiments are expressed as means ( \pm S.D.). Unpaired Student's $t$-tests were used to analyze between-group differences that are repeated and $P$-value $<0.05$ was considered as statistically significant.

\section{Conflict of Interest}

The authors declare no conflict of interest.

Acknowledgements. This work was supported by grants from National Natural Science Foundation of China (31070729, 31270866, and 31200606), Major State Basic Research Development Program of China (2012CB822103), Specialized Research Fund for the Doctoral Program of Higher Education of China (20122105120003), and National Natural Science Foundation of Liaoning (2014023045).

1. Guzeloglu-Kayisli O, Basar M, Arici A. Basic aspects of implantation. Reprod Biomed Online 2007; 15: 728-739.

2. Bettegowda A, Smith GW. Mechanisms of maternal mRNA regulation: implications for mammalian early embryonic development. Front Biosci 2007; 12: 3713-3726.

3. Red-Horse K, Zhou Y, Genbacev O, Prakobphol A, Foulk R, McMaster M et al. Trophoblast differentiation during embryo implantation and formation of the maternal-fetal interface. $J$ Clin Invest 2004; 114: 744-754.

4. Lunghi L, Ferretti ME, Medici S, Biondi C, Vesce F. Control of human trophoblast function Reprod Biol Endocrinol 2007; 8: 5-19.

5. Castellucci M, Kosanke G, Verdenelli F, Huppertz B, Kaufmann P. Villous sprouting: fundamental mechanisms of human placental development. Hum Reprod Update 2000; 6 : 485-494.

6. Loregger T, Pollheimer J, Knöfler M. Regulatory transcription factors controlling function and differentiation of human trophoblast-a review. Placenta 2003; 24(Suppl A): S104-S110.

7. Emiliani S, Delbaere A, Devreker F, Englert Y. Embryo-maternal interactive factors regulating the implantation process: implications in assisted reproductive. Reprod Biomed Online 2005; 10: 527-540.

8. Dey SK, Lim H, Das SK, Reese J, Paria BC, Daikoku T et al. Molecular cues to implantation. Endocr Rev 2004; 25: 341-373.

9. Cheng JC, Chang HM, Leung PC. Transforming growth factor- $\beta 1$ inhibits trophoblast cell invasion by inducing Snail-mediated down-regulation of vascular endothelial-cadherin protein. J Biol Chem 2013; 288: 33181-33192.

10. He P, Srikrishna G, Freeze HH. N-glycosylation deficiency reduces ICAM-1 induction and impairs inflammatory response. Glycobiology 2014; 24: 392-398.

11. Croci DO, Cerliani JP, Dalotto-Moreno T, Méndez-Huergo SP, Mascanfroni ID Dergan-Dylon $S$ et al. Glycosylation-dependent lectin-receptor interactions preserve angiogenesis in anti-VEGF refractory tumors. Cell 2014; 156: 744-758.

12. Jones CJ, Aplin JD. Glycosylation at the fetomaternal interface: does the glycocode play a critical role in implantation? Glycoconj J 2009; 26: 359-366.

13. Tu Z, Lin YN, Lin CH. Development of fucosyltransferase and fucosidase inhibitors. Chem Soc Rev 2013; 42: 4459-4475.

14. Becker DJ, Lowe JB. Fucose: biosynthesis and biological function in mammals. Glycobiology 2003; 13: 41-53.

15. Aplin JD, Jones CJ. Fucose placental evolution and the glycocode. Glycobiology 2012; 22: $470-478$.

16. Orczyk-Pawiłowicz M, Augustyniak D, Hirnle L, Katnik-Prastowska I. Lectin-based analysis of fucose and sialic acid expressions on human amniotic IgA during normal pregnancy. Glycoconj J 2013; 30: 599-608.

17. Ponnampalam AP, Rogers PA. Expression and regulation of fucosyltransferase 4 in human endometrium. Reproduction 2008; 136: 117-123.

18. Liu S, Yang X, Wang J, Wei J, Zhang D, Wang X et al. Differential expression of LeY and fucosyltransferase IV correlates with the receptivity of RL95-2 and HEC-1A human uterine epithelial cells. Cell Biol Int 2012; 36: 469-474.

19. Liu S, Zhang Y, Liu Y, Qin H, Wang X, Yan Q. FUT7 antisense sequence inhibits the expression of FUT7/sLeX and adhesion between embryonic and uterine cells. IUBMB Life 2008; 60: 461-466.

20. Shi S, Stanley P. Protein O-fucosyltransferase 1 is an essential component of Notch signaling pathways. Proc Natl Acad Sci 2003; 100: 5234-5239.

21. Yamakawa T, Yamada K, Sasamura T, Nakazawa N, Kanai M, Suzuki E et al. Deficient Notch signaling associated with neurogenic pecanex is compensated for by the unfolded protein response in Drosophila. Development 2012; 139: 558-567. 
22. Loriol C, Dupuy F, Rampal R, Dlugosz MA, Haltiwanger RS, Maftah A etal. Molecular evolution of protein O-fucosyltransferase genes and splice variants. Glycobiology 2006; 16: 736-747.

23. Du J, Takeuchi H, Leonhard-Melief C, Shroyer KR, Dlugosz M, Haltiwanger RS et al. O-fucosylation of thrombospondin type 1 repeats restricts epithelial to mesenchymal transition (EMT) and maintains epiblast pluripotency during mouse gastrulation. Dev Biol 2010; 346: 25-38.

24. Dimitriadis E, Menkhorst E, Salamonsen LA, Paiva P. Review: LIF and IL11 in trophoblastendometrial interactions during the establishment of pregnancy. Placenta 2010; 31: S99-S104.

25. Suman P, Malhotra SS, Gupta SK. LIF-STAT signaling and trophoblast biology. JAKSTAT 2013; 2: e25155.

26. Cullinan EB, Abbondanzo SJ, Anderson PS, Pollard JW, Lessey BA, Stewart CL. Leukemia inhibitory factor (LIF) and LIF receptor expression in human endometrium suggests a potential autocrine/paracrine function in regulating embryo implantation. Proc Natl Acad Sci USA 1996; 93: 3115-3120.

27. Fitzgerald JS, Poehlmann TG, Schleussner E, Markert UR. Trophoblast invasion: the role of intracellular cytokine signalling via signal transducer and activator of transcription 3 (STAT3). Hum Reprod Update 2008; 14: 335-344.

28. Sharkey AM, King A, Clark DE, Burrows TD, Jokhi PP, Charnock-Jones DS et al Localization of leukemia inhibitory factor and its receptor in human placenta throughout pregnancy. Biol Reprod 1999; 60: 355-364.

29. Cheng JG, Chen JR, Hernandez L, Alvord WG, Stewart CL. Dual control of LIF expression and LIF receptor function regulate Stat3 activation at the onset of uterine receptivity and embryo implantation. Proc Natl Acad Sci USA 2001; 98: 8680-8685.

30. Li W, Liu D, Chang W, Lu X, Wang YL, Wang H et al. Role of IGF2BP3 in trophoblast cell invasion and migration. Cell Death Dis 2014; 5: e1025.

31. Zhang Q, Chen Q, Lu X, Zhou Z, Zhang H, Lin HY et al. CUL1 promotes trophoblast cell invasion at the maternal-fetal interface. Cell Death Dis 2013; 4: e502.

32. Hromatka BS, Drake PM, Kapidzic M, Stolp H, Goldfien GA, Shih leM et al. Polysialic acid enhances the migration and invasion of human cytotrophoblasts. Glycobiology 2013; 23: 593-602.

33. Yamamoto E, Ino K, Miyoshi E, Inamori K, Abe A, Sumigama S et al. N-acetylglucosaminyltransferase $\mathrm{V}$ regulates extravillous trophoblast invasion through glycosylation of alpha5beta1 integrin. Endocrinology 2009; 150: 990-999.

34. Prakash GJ, Suman P, Morales Prieto DM, Markert UR, Gupta SK. Leukaemia inhibitory factor mediated proliferation of HTR-8/SVneo trophoblast cells is dependent on activation of extracellular signal-regulated kinase 1/2. Reprod Fertil Dev 2011; 23: 714-724.

35. Mariee N, Li TC, Laird SM. Expression of leukaemia inhibitory factor and interleukin 15 in endometrium of women with recurrent implantation failure after IVF; correlation with the number of endometrial natural killer cells. Hum Reprod 2012; 27: 1946-1954.

36. Krishnan T, Winship A, Sonderegger S, Menkhorst E, Horne AW, Brown J et al. The role of leukemia inhibitory factor in tubal ectopic pregnancy. Placenta 2013; 34: 1014-1019.

37. Takahashi Y, Takahashi M, Carpino N, Jou ST, Chao JR, Tanaka S et al. Leukemia inhibitory factor regulates trophoblast giant cell differentiation via Janus kinase 1-signal transducer and activator of transcription 3-suppressor of cytokine signaling 3 pathway. $\mathrm{Mol}$ Endocrinol 2008; 22: 1673-1681.

38. Suman P, Shembekar N, Gupta SK. Leukemia inhibitory factor increases the invasiveness of trophoblastic cells through integrated increase in the expression of adhesion molecules and pappalysin 1 with a concomitant decrease in the expression of tissue inhibitor of matrix metalloproteinases. Fertil Steril 2013; 99: 533-542.
39. Zhao M, Chang C, Liu Z, Chen LM, Chen Q. Treatment with low-dose aspirin increased the level LIF and integrin $\beta 3$ expression in mice during the implantation window. Placenta 2010; 31: 1101-1105.

40. Bamberger AM, Jenatschke S, Schulte HM, Löning T, Bamberger MC. Leukemia inhibitory factor (LIF) stimulates the human HLA-G promoter in JEG3 choriocarcinoma cells. J Clin Endocrinol Metab 2000; 85: 3932-3936.

41. Zhang Q, Liu S, Zhu Z, Yan Q. Regulating effect of LIF on the expression of FuT7: Probe into the mechanism of sLe(x) in implantation. Mol Reprod Dev 2009; 76: 692.

42. Staun-Ram E, Shalev E. Human trophoblast function during the implantation process. Reprod Biol Endocrinol 2005; 20: 56.

43. Zhang H, Hou L, Li CM, Zhang WY. The chemokine CXCL6 restricts human trophoblast cell migration and invasion by suppressing MMP-2 activity in the first trimester. Hum Reprod 2013; 28: 2350-2362.

44. Sonderegger S, Haslinger P, Sabri A, Leisser C, Otten JV, Fiala C et al. Wingless (Wnt)-3A induces trophoblast migration and matrix metalloproteinase-2 secretion through canonical Wnt signaling and protein kinase B/AKT activation. Endocrinology 2010; 151: 211-220.

45. Baker AH, Edwards DR, Murphy G. Metalloproteinase inhibitors: biological actions and therapeutic opportunities. J Cell Sci 2002; 115: 3719-3727.

46. Leduc K, Bourassa V, Asselin E, Leclerc P, Lafond J, Reyes-Moreno C. Leukemia inhibitory factor regulates differentiation of trophoblastlike BeWo cells through the activation of JAK/STAT and MAPK3/1 MAP kinase-signaling pathways. Biol Reprod 2012; 29: 54

47. Hirai H, Karian P, Kikyo N. Regulation of embryonic stem cell self-renewal and pluripotency by leukaemia inhibitory factor. Biochem $J 2011 ; 438$ : 11-23.

48. Chen L, Khillan JS. A novel signaling by vitamin A/retinol promotes self renewal of mouse embryonic stem cells by activating PI3K/Akt signaling pathway via insulin-like growth factor-1 receptor. Stem Cells 2010; 28: 57-63.

49. Yang X, Liu S, Yan Q. Role of fucosyltransferase IV in epithelial-mesenchymal transition in breast cancer cells. Cell Death Dis 2013; 4: e735.

50. Cheng JC, Chou $\mathrm{CH}$, Kuo ML, Hsieh CY. Radiation-enhanced hepatocellular carcinoma cell invasion with MMP-9 expression through PI3K/Akt/NF-kappaB signal transduction pathway. Oncogene 2006; 25: 7009-7018.

51. Genbacev O, Jensen KD, Powlin SS, Miller RK. In vitro differentiation and ultrastructure of human extravillous trophoblast (EVT) cells. Placenta 1993; 14: 463-475.

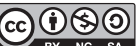

Cell Death and Disease is an open-access journal published by Nature Publishing Group. This work is licensed under a Creative Commons Attribution-NonCommercialShareAlike 3.0 Unported License. The images or other third party material in this article are included in the article's Creative Commons license, unless indicated otherwise in the credit line; if the material is not included under the Creative Commons license, users will need to obtain permission from the license holder to reproduce the material. To view a copy of this license, visit http://creativecommons.org/licenses/ by-nc-sa/3.0/ 